\title{
AM FM based Prediction of Multiple Sclerosis in Brain MRI Images
}

\author{
S.P.Washimkar \\ Asst. Professor \\ Electronics \& Telecommunication Engg Dept. PCE, \\ Nagpur
}

\author{
S.D.Chede, Ph.D \\ Principal \\ Om college of Engg., Wardha
}

\begin{abstract}
White matter is one of the two components of central nervous system and consists mostly of glialcells and myelinated axons that transmits signal from one region of the cerebrum to another and between cerebrum and lower brain centers. Multiple sclerosis (MS) is one of the most common diseases which affect white matter. Multiple sclerosis is a chronic idiopathic disease resulted in multiple areas of inflammatory demyelization in the Central nervous system. MS lesion formation often leads to unpredictable cognitive decline \& Physical disability. Due to the sensitivity in detecting MS lesions, MRI has become an important tool for diagnosing MS \& monitoring its progression. Radiological criteria for MS include the number of lesions (some scattered bright spot) on the MRI, their location and their size. Due to the complexity \& variance of automated MRI segmentation of brain MS became a complex task. A structural texture analysis method on MS segmentation scheme gives emphasis on structural analysis of MS as well as on normal tissues. An important tool that has been developed and used in variety of research is the Image Modulation model, also termed the AmplitudeModulation, Frequency-Modulation (AM-FM) image model, which models non-stationary image content using an AM-FM expansion. The AM-FM technique offers advantages for feature extraction at different frequency scales and orientations that can be used to detect different patterns, directions, or structures in an image. Thus high-frequency scale instantaneous amplitude can be used to differentiate between lesions associated with early and advanced disease stages and thus AM-FM technique can offer excellent results in classification of Multiple sclerosis from the white matter of the nervous system.
\end{abstract}

\section{Keywords}

AM FM, Multiple Sclerosis (MS), Magnetic Resource Image (MRI), K-Nearest Neighbor Algorithm (KNN), Support Vector Machine (SVM), GA, PSO.

\section{INTRODUCTION}

Multiple sclerosis is an autoimmune disease that affects the brain and spinal cord (central nervous system)[1][2].Multiple sclerosis affects women more than men. The disorder is most commonly diagnosed between ages 20 and 40 , but can be seen at any age. MS is caused by damage to the myelin sheath, the protective covering that surrounds nerve cells as shown in fig. 1,2 . When this nerve covering is damaged, nerve signals slow down or stop. The nerve damage is caused by inflammation. Inflammation occurs when the bodies own immune cells attack the nervous system. This can occur along any area of the brain, optic nerve, and spinal cord. It is unknown what exactly causes this to happen. The most common thought is that a virus or genetic defect, or both, is to blame. Environmental factors may play a role. You are slightly more likely to get this condition if you have a family history of MS or live in a part of the world where MS is more common. Thorough medical history and exam, a variety of specialized procedures are helpful although not always necessary to accurately diagnose MS. These include imaging techniques, such as MRI, spinal taps or lumbar punctures (examination of the cerebrospinal fluid that runs through the spinal column), evoked potentials (electrical tests to help determine if MS has affected a person's nerve pathways), and lab analysis of blood samples.

MRI is the best test to view the changes caused by multiple sclerosis .The precise image produced by MRI gives the neurologist clear evidence of scar tissue in the deep parts of the brain or spinal cord that is characteristic of MS. However, abnormal spots on the brain MRI can be caused by other conditions, so before making a diagnosis your doctor will consider all information including your symptoms and scan results. Similar lesions can be seen in elderly people or people with migraine headaches or high blood pressure. Also, a normal MRI does not absolutely rule out a diagnosis of MS. About $5 \%$ of patients, who are confirmed to have MS on the basis of other criteria, do not have lesions in the brain on MRI. These people may have lesions in the spinal cord or may have lesions that cannot be detected by MRI.

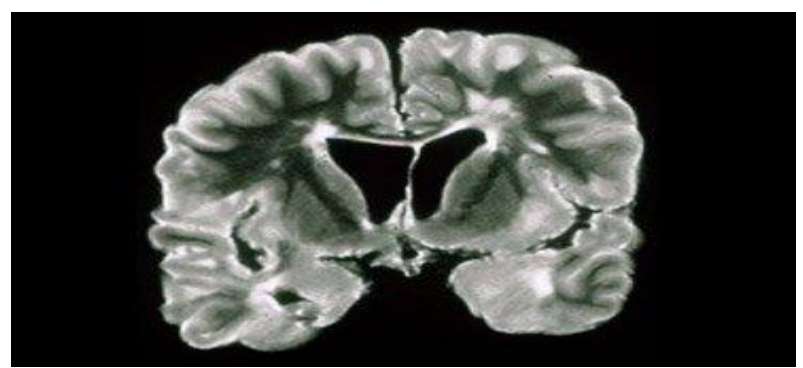

Figure 1. Coronal PD image of a brain specimen with MS involvement

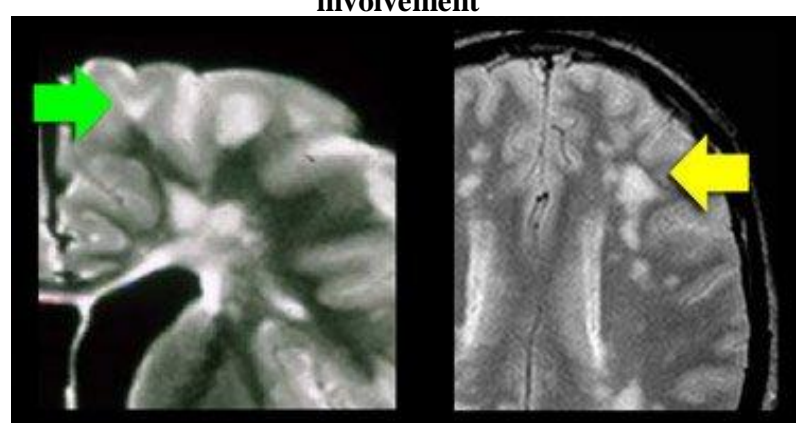

Figure 2. LEFT - Involvement of U fibers in MS RIGHT $\mathrm{U}$ fibers are not involved in patient with hypertension 
The Fazekas classification is used to describe changes in the deep white matter. Whenever you see these White Matter Lesion WMLs, try to describe them according to this Fazekas classification .[8]

Fazekas I: small punctuate lesions in the deep white matter - considered normal in aging.

Fazekas II: larger WMLs that are beginning to become confluent considered abnormal in patients $<75$ Years.

\section{Fazekas III: extensive confluent WMLs - abnormal in any age group}

These WMLs are probably due to microangiopathy and seen more frequently in patients with vascular risk factors.

\subsection{AM-FM}

Many feature extraction techniques had been widely used by different researchers in wide variety of applications such as Principal Component Analysis, Independent Component Analysis, Wavelet Transform, and statistical analysis. For texture analysis AM-FM modulation represents an emerging technique that shows great promise in this area. Multidimensional AM-FM models and methods provide us with powerful, image and video decompositions that can effectively describe non-stationary content. They represent an extension to standard Fourier analysis, allowing both the amplitude and the phase functions to vary spatially over the support of the image, following changes in local texture and brightness. AM-FM decompositions provide physically meaningful texture measurements. Significant texture variations are captured in the frequency components. For single component cases, IF vectors are orthogonal to equiintensity lines of an image, while the IF magnitude provides a measure of local frequency content.

Since AM-FM texture features are provided at a pixel-level resolution, AM-FM models can be used to segment texture images that are difficult to model with the standard brightness-based methods [4]. On the other hand, using just histograms of the IF and Instantaneous Amplitude (IA), we can design effective content-based image retrieval systems using very short image feature vectors [6]].In the past few years, as the understanding of its theory advanced, AM-FM decompositions have been applied in a series of medical imaging problems ranging from ultrasound to retinal image analysis, yielding excellent results. In summary, the advantages of AM-FM methods include i) they provide a large number of physically meaningful texture features, over multiple scales, at a pixel-level resolution, (ii) the image can be reconstructed from the AM-FM decompositions, (iii) based on the target application, different AM-FM decompositions using different frequency coverage can be designed [7].

\subsubsection{The AM-FM Method}

In this paper the Amplitude-modulation frequencymodulation (AM-FM) decompositions represent images using spatially-varying sinusoidal waves and their spatially-varying amplitudes. AM-FM decompositions use different scales and band pass filters to extract the wide range of instantaneous frequencies and instantaneous amplitude components that may be present in an image as shown in fig.3 [7].

\subsubsection{The basic AM-FM model}

In the 2D model, we expand an input image $I(x, y)$ into a sum of AM-FM harmonics using:

$$
\mathrm{I}(x, y)=\sum_{n=1}^{\mathrm{M}}\left(a_{n}(x, y) \cos \varphi n(x, y)\right)---1
$$

Where, $a_{n}(x, y) \quad$ denote slowly-varying instantaneous amplitude (IA) functions, $\varphi_{n}(x, y)$ denote the instantaneous phase (IP) components, and $n=1,2, \ldots, M$ indexes the different AM-FM harmonics. In above equation the $n$th AMFM harmonic is represented by $a_{n}(x, y) \cos \varphi_{n}(x, y)$. With each phase function, the instantaneous frequency (IF) vector field is defined by

$$
\nabla \emptyset n(x, y)=\left(\frac{\partial \emptyset n}{\partial x}(x, y), \frac{\partial \emptyset n}{\partial y}(x, y)\right) \quad--2
$$

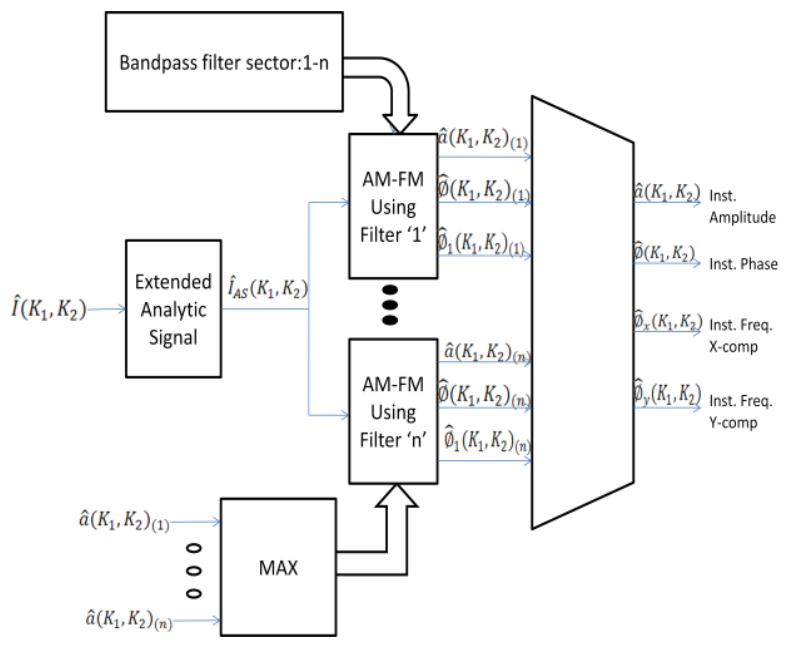

Figure3. Block diagram of the 2D multi scale AM-FM demodulation.

Here, the AM-FM demodulation problem is defined as one of determining the IA, IP, and IF functions for any given input image. The figure shows how these components are used to determine the IA, IP and IF components. In the equation $n=1$, $2,3 \ldots . . . M$ denotes AM-FM components from different scales using dominant component analysis. The term scales is collection of low, medium and high frequency components. To improve IA estimation, optimal designed digital filters are used. They allow controlling the pass band gain close to 1 and stopping band gain closer to 0 . Hence the digital filters remove the IA correction step.[3] 


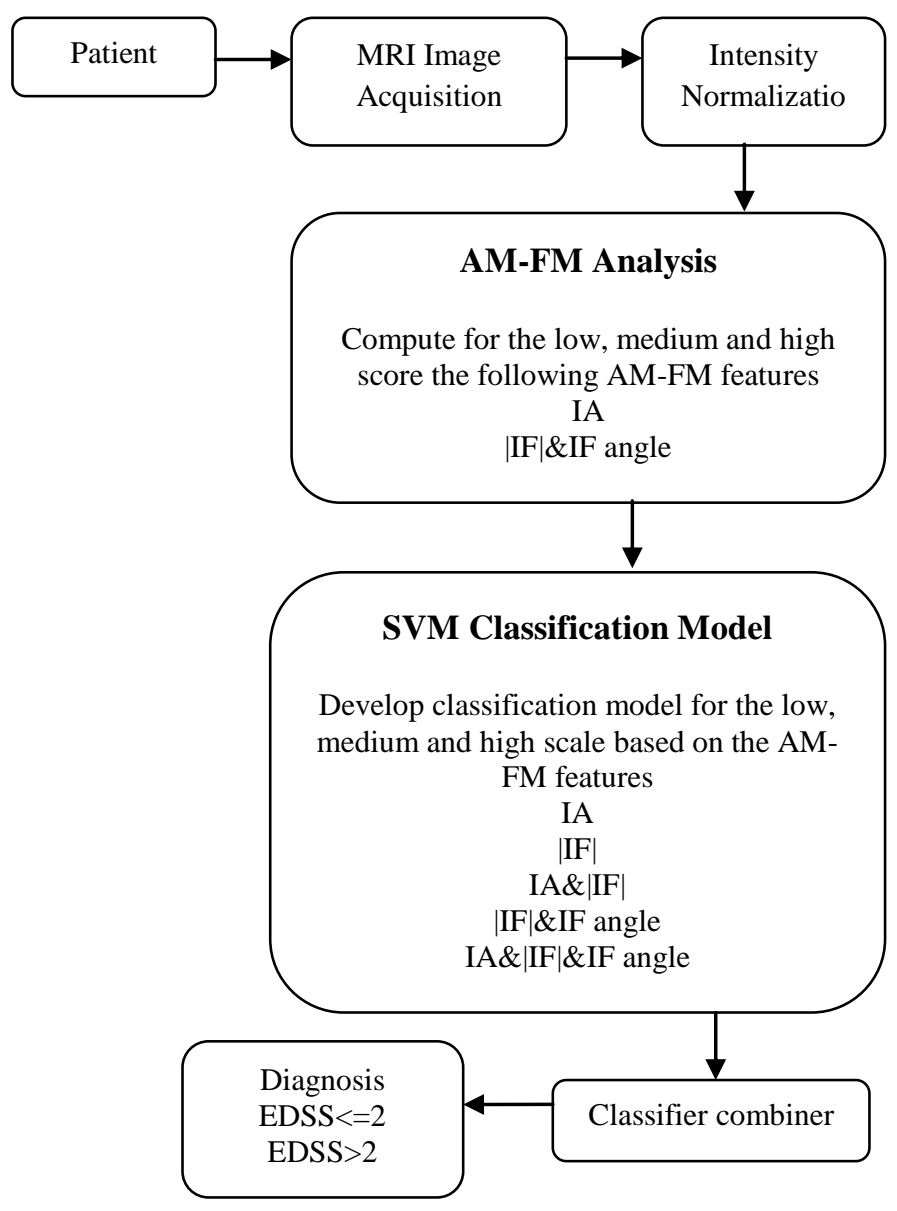

Figure4. MRI-image-analysis system diagram.

Initial diagnosis was made by an experienced neurologist who referred patient for a baseline MRI upon diagnosis and followed patient for two years. Patient remains untreated between baseline MRI and repeat MRI.At the initial scan the stage of the disease was evaluated using EDSS score.[4]

All detectable brain lesions were identified and segmented by an experienced MS neurologist and confirmed by radiologist. The manual delineation was performed using GUI Implemented in matlab. From MRI scan, ROI were selected. The selected points and delineations were saved to be used for texture analysis.Interscan Intensity normalization method was used on MRI scan.AM-FM Segmented image was obtained using GUI implemented in matlab. Features were extracted from AM-FM segmented image. A classification model was developed to classify normal image and diseased image or to see the progression of disease for successive stages

\section{ALGORITHM}

\subsection{K Nearest Neighbour Algorithm}

$\mathrm{K}$ nearest neighbor algorithm is also called as lazy learning algorithm. This is so because it defers the decision to generalize till a new query is encountered. Whenever we have a new point to classify, we find its $\mathrm{K}$ nearest neighbors from the training data [9].

1) For each training example $\langle x f(x)\rangle$, add the example to the list of training examples

2) Given a query instance $x_{q}$ to be classified a) Let $\mathrm{x} 1, \mathrm{x} 2 \ldots \ldots \ldots$. denote the $\mathrm{k}$ instances from training examples that are nearest to $x_{q}$.

b) Return the class that represents the maximum of the $\mathrm{k}$ instances.

\section{PROPOSD METHOD}

The overall diagrammatic representation of the proposed work is as shown below:-

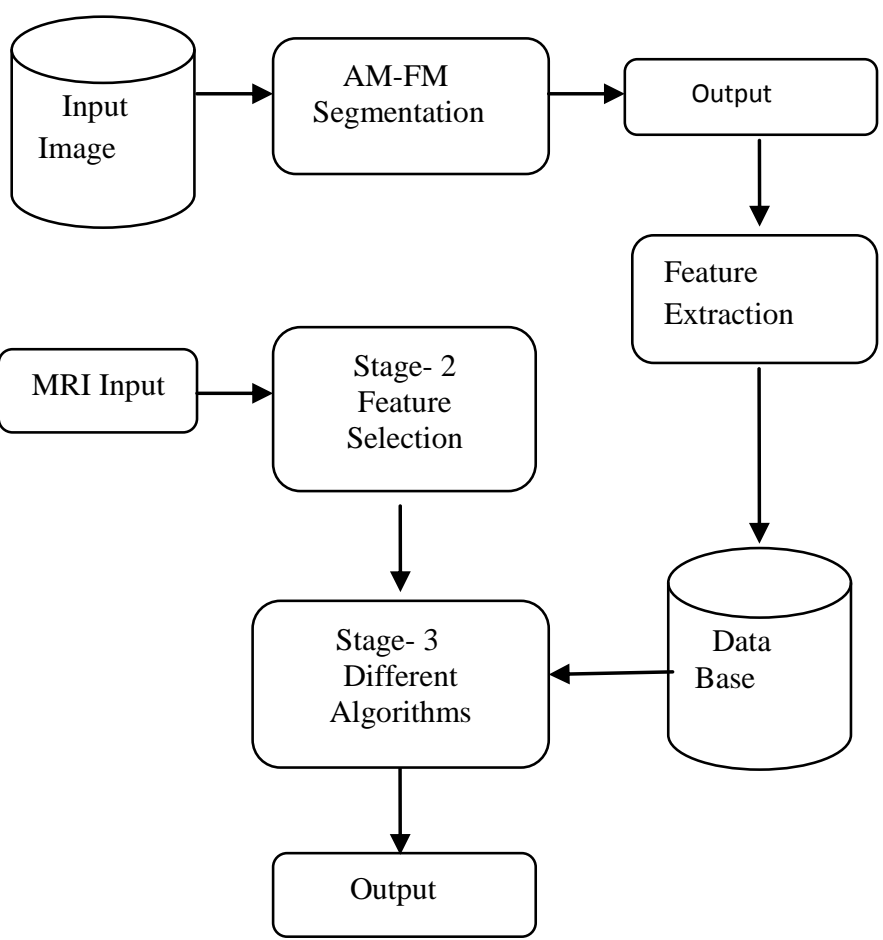

Figure5. Diagrammatic representation of work flow

The proposed methodology consists of two phases:-

1) Training Phase and

2) Evaluation Phase

\subsection{Training Phase}

In training phase firstly the complete system is trained the database. The MRI image of the brain is taken as the input for the methodology. AM-FM Segmentation is used for the image processing. The output image from the AM-FM segmentation is used for getting the features from the image. Features like Area of the image, Eccentricity of the MRI image, Maximum axis, minimum axis of the MRI image, texture, color, shape and orientation feature of the AM-FM segmentation output image. This features the store in the database with the name of the disease for the evaluation phase.

\subsection{Evaluation Phase}

In the evaluation phase firstly features of the MRI image is calculated and various algorithms are applied to find the best method for diseases detection of the brain.KNN, SVM, POS and GA algorithm are used for the evaluation method. Now the features of the IMR are given as input to the system as shown below with the database we previously created and apply the following algorithms for the comparison of the output:- 


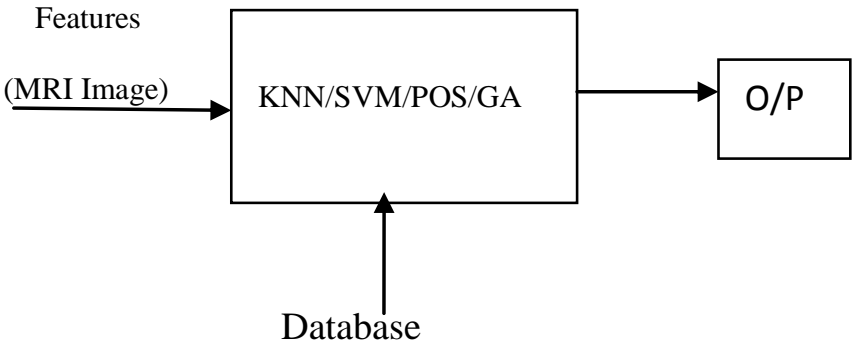

Figure6. Diagrammatic Representation of Evaluation Phase

Now the features are given as input to the system. Here $\mathrm{KNN} / \mathrm{SVM} / \mathrm{POS} / \mathrm{GA}$ algorithms are applied to the input which gives the output as a class of disease. These also gives the periods of the treatment needed for the disease.

\section{RESULT}

Table 1 shows the evaluation of the MRI images of the two different patients using KNN (K- Nearest Neighbor) method. As the table shows the accuracy of KNN algorithm to predict the week from the MRI image for 0 week, 24 week and 144 week is $100 \%$. We tested $60,15,15$ and 15 for $0^{\text {th }}$ week, $24^{\text {th }}$ week, $96^{\text {th }}$ week and $144^{\text {th }}$ week respectively.

\section{Table 1 Evaluation Result from KNN Algorithm}

\begin{tabular}{|c|c|c|c|}
\hline Patient & $\begin{array}{l}\text { No. } \\
\text { Weeks }\end{array}$ & $\begin{array}{l}\text { Total No. } \\
\text { of images } \\
\text { Tested }\end{array}$ & Accuracy \\
\hline 1 & 0 & 60 & $100 \%$ \\
\hline 1 & 24 & 15 & $100 \%$ \\
\hline 1 & 96 & 15 & Less than 5\% \\
\hline 1 & 144 & 15 & $100 \%$ \\
\hline 2 & 0 & 60 & $100 \%$ \\
\hline 2 & 24 & 15 & $100 \%$ \\
\hline 2 & 96 & 15 & Less than 5\% \\
\hline 2 & 144 & 15 & $100 \%$ \\
\hline
\end{tabular}

But from the table 1 it shown that the evaluation result for the MRI images of $96^{\text {th }}$ week is very low i.e. the accuracy of the $96^{\text {th }}$ week image is less than $5 \%$. So in our proposed method we are going to improve the accuracy of the $96^{\text {th }}$ week MRI image meanwhile maintaining the accuracy of the other weeks evaluation of the KNN algorithm

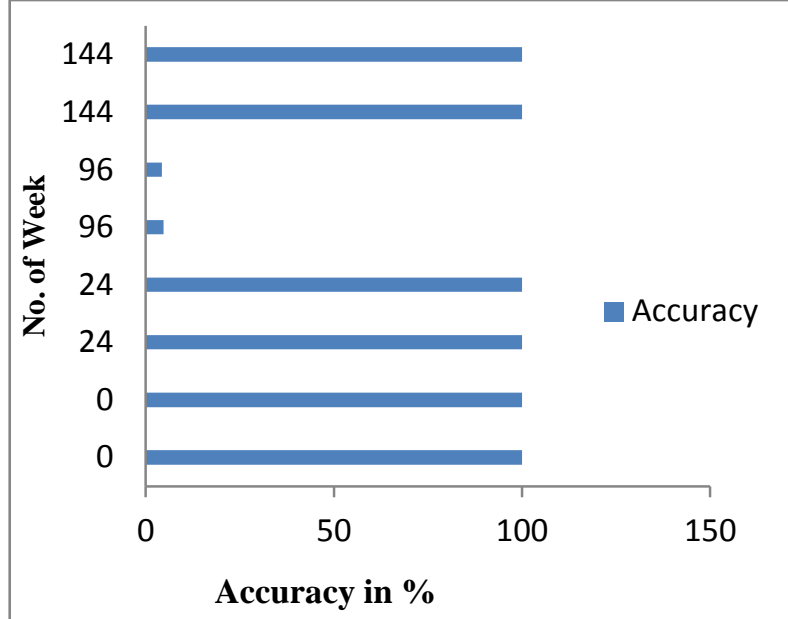

Figure7. Bar Graph of the Evaluation Result from KNN Algorithm

\section{CONCLUSION}

MRI analysis has become a powerful tool in the diagnosis of brain disease. Pixel intensity variations between the same and consecutive MRI scans i.e. intra-scan and inter-scan variations, complicate the method of quantitative MRI analysis. Improvements in the measurement and preprocessing of the image may therefore have a significant impact in the clinical diagnosis, image analysis, and computer aided diagnosis. In the current case study of MS patients, it was shown that the simple AM-FM method of histogram intensity normalization proposed can help in this direction. This will enable a more accurate computation of texture features that may provide information for the earlier differentiation between normal tissue and MS lesions and in assessing disease progression

\section{REFERENCES}

[1] F. Fazekas, F. Barkof, M. Filippi, et al, "The contribution of magnetic resonance imaging to the diagnosis of multiple sclerosis," Neur., vol.53, pp.448-456, 1999.

[2] M. Filippi, D.W. Paty, L. Kappos, F. Barkhof, D.A. et. aI., "Correlation between changes in disability and T2weighted brain MRI activity in multiple sclerosis: a follow-up study," Neur., vol. 45, pp.255-260, 1995

[3] Victor Murray, Paul Rodriguez, and Marios S. Pattichis,"Multiscale AM-FM Demodulation and Image Reconstruction Methods With Improved Accuracy", IEEE Transactions on image processing, v1 9 no. 5, may 2010 .

[4] C.P. Loizou, V. Murray, M.S. Pattichis, I Seimenis ,M Pantziaris,C.S Pattichis," Multiscale AmplitudeModulation Frequency Modulation(AM-FM) Tecture analysis of Multiple Sclerosis in Brain MRI Images". IEEETransactions on information technology in biomedicine, vol 15,no.1,january 2011

[5] J. Dehmeshki, G. J. Barker and P. S. Tofts," Classification of Disease Subgroup and Correlation with Disease Severity Using Magnetic Resonance Imaging Whole-BrainHistograms:ApplicationtoMagnetization Transfer Ratios and Multiple Sclerosis", IEEE transactions on Medical Imaging, vol. 21, no. 4, April 2002. 
[6] Victor Murray, Marios S Pattichis, Eduardo S Barriga and Peter Soliz," Recent multiscale AM-FM methods in emerging applications in medical imaging", EURASIP Journal on Advances in Signal Processing 2012.

[7] Victor Murray, Eduardo S. Barriga, Peter Soliz and Marios S. Pattichis," Survey of AM-FM methods for Applications in Medical Imaging".
[8] Fazekas (Graz)," The contribution of magnetic resonance imaging to the diagnosis of multiple sclerosis", $43^{\text {rd }}$ International Neuropsychiatric PULA Symposium

[9] I. Guyon and A. Elisseeff, An Introduction to Variable and Feature Selection J. Machine Learning Research, vol. 3, pp. 1157-1182, 2003. 